ARTIGO ORIGINAL

\title{
Configuração de redes neurais artificiais para relação hipsométrica de árvores de Eucalyptus spp.
}

\author{
Configuration of artificial neural networks for height-diameter \\ relationship of Eucalyptus spp.
}

Jonas Elias Castro da Rocha ${ }^{1}$ (D), Marlon Roque Nogueira Junior ${ }^{1}$ (D), Ivaldo da Silva Tavares Júnior ${ }^{2}$ (D), Jianne Rafaela Mazzini de Souza² (1), Lucas Sérgio de Sousa Lopes² (1), Márcio Lopes da Silva² (1)

'Universidade Federal Rural da Amazônia - UFRA, Paragominas, PA, Brasil

${ }^{2}$ Universidade Federal de Viçosa - UFV, Viçosa, MG, Brasil

Como citar: Rocha, J. E. C., Nogueira Junior, M. R., Tavares Júnior, I. S., Souza, J. R. M., Lopes, L. S. S., Silva, M. L. (2021). Configuração de redes neurais artificiais para relação hipsométrica de árvores de Eucalyptus spp. Scientia Forestalis, 49(132), e3706. https://doi.org/10.18671/scifor.v49n132.08

\begin{abstract}
Resumo
Este estudo explora algoritmos e funções de ativação de redes neurais artificiais (RNAs) para a predição da altura total das árvores de Eucalyptus spp. O objetivo foi recomendar as melhores configurações de RNAs para essa variável. Os dados foram provenientes de 2.888 árvores. As RNA treinadas apresentaram como variáveis de entrada o DAP, clone, idade, classe de idade e classe diamétrica. A altura total foi a variável de saída. Cinco algoritmos e seis funções de ativação foram combinados nas camadas oculta e de saída, totalizando 18.125 RNA treinadas. As RNA foram avaliadas por meio da correlação linear $\left(r_{\hat{y} y}\right)$, raiz quadrada do erro médio (RQEM\%), bias e histogramas da $r_{\hat{y} y}$ e RQEM\%. As RNAs treinadas obtiveram RQEM\% variando de $0,07 \%$ a 396,3\% e $r_{\hat{y} y}$ de $-0,7130$ a 0,9998. O treinamento das RNAs foi realizado no software Neuro 4.0.6. Com exceção da RNA com o algoritmo Manhattan Update Rule, as melhores RNA selecionadas na validação apresentaram correlação acima de 0,97, e RQEM\% e bias próximos de zero. Os algoritmos Backpropagation, Resilient Propagation, Scaled Conjugate Gradient e Quick Propagation apresentaram boa precisão na modelagem da altura. As funções de ativação logística e log são eficientes para as camadas oculta e de saída, respectivamente. Na validação, a topologia de rede 12-10-1 com algoritmo Resilient Propagation apresentou a maior precisão, com RQEM de 0,067 m. Por outro lado, a topologia 12-14-1 com algoritmo Manhattan Update Rule obteve a menor precisão, com RQEM de 3,13 m. A topologia 12-10-1, com algoritmo Resilient Propagation e função de ativação logística, podem ser utilizadas no treinamento para a predição da altura total de Eucalyptus spp.
\end{abstract}

Palavras-chave: Altura; Eucalipto; Inteligência artificial; Manejo florestal.

\begin{abstract}
This study explores algorithms and functions of activation of artificial neural networks (ANNs) to predict the total height of Eucalyptus spp. The objective was to recommend the best RNA configurations for this variable. The data came from 2,888 trees. The trained ANNs presented $\mathrm{DBH}$, clone, age, age class and diametric class as input variables. The total height was the output variable. Five algorithms and six activation functions were combined in the hidden and output layers, totaling 18,125 trained ANNs. ANNs were evaluated using linear correlation $\left(r_{\hat{y} y}\right)$, square root of the average error (RMSE\%), bias and histograms of $r_{\hat{y} y}$ and RMSE\%. The trained ANNs obtained RMSE\% ranging from $0.07 \%$ to $396.3 \%$ and $r_{\hat{y} y}$ of -0.7130 to 0.9998 . The ANNs was performed using the Neuro 4.0.6 software. With the exception of ANN with the Manhattan Update Rule algorithm, the best ANN selected in the validation showed a
\end{abstract}

Fonte de financiamento: Nada a declarar.

Conflito de interesse: Nada a declarar.

Autor correspondente: jianne.souza@ufv.br

Recebido: 27 abril 2021.

Aceito: 24 maio 2021.

Editor: Paulo Henrique Müller Silva. 
correlation above 0.97, and RMSE\% and bias close to zero. The Backpropagation, Resilient Propagation, Scaled Conjugate Gradient and Quick Propagation algorithms presented satisfactory results in height modeling. The logistic and log activation functions are efficient for the hidden and output layers, respectively. In validation, the 12-10-1 network architecture with a Resilient Propagation algorithm showed the highest precision, with RMSE of $0.067 \mathrm{~m}$. On the other hand, the architecture 12-14-1 with the Manhattan Update Rule algorithm resulted in the lowest precision, with RMSE of $3.13 \mathrm{~m}$. The 12-10-1 network architecture, with Resilient Propagation algorithm and logistical activation function, can be used in the training for the prediction of the total height of Eucalyptus spp.

Keywords: Height; Eucalyptus; Artificial intelligence; Forest management.

\section{INTRODUÇÃO}

A predição do crescimento de Eucalyptus spp. é imprescindível para o manejo florestal, uma vez que essa predição fornece informações quantitativas sobre a floresta, corroborando para o planejamento e análises econômicas (Binoti et al., 2014). Para tanto, são comumente aplicados inventários florestais contínuos (IFC) para avaliar o desenvolvimento do povoamento.

Contudo, o IFC demanda tempo e custos, pois algumas variáveis são de difícil obtenção, como por exemplo a altura das árvores, que é mensurada indiretamente com auxílio de aparelhos hipsométricos (Campos \& Leite, 2017). Mesmo que esses aparelhos tenham evoluído quanto a sua acuracidade, ainda estão sujeitos a erros devido à falta de visibilidade do topo da árvore (Cysneiros et al., 2020). Dessa forma, torna-se comum utilizar métodos baseados em relações hipsométricas para predizer essa variável, de forma a reduzir tempo e capital nos IFC (Binoti et al., 2013; Melo et al., 2017). Dentre as diversas técnicas utilizadas para este fim, as redes neurais artificiais (RNA) vêm se destacando como uma técnica eficiente para predizer variáveis importantes em um inventário florestal.

As RNA já foram bem aplicadas na predição de diversas variáveis florestais, como do volume de árvores (Özçelik et al., 2010), biomassa acima do solo (Nandy et al., 2017), densidade da madeira (Demertzis et al., 2017) e inclusive da altura de árvores (Vieira et al., 2018). Contudo, apesar da grande quantidade de estudos na literatura com essa técnica, as aplicações na região amazônica ainda são raras. Além disso, o desempenho de uma RNA na predição da altura das árvores pode ser otimizado com a escolha de uma função de ativação e algoritmo adequado, que pode diminuir a complexidade da rede e aumentar o desempenho (Vendruscolo et al., 2017).

Os algoritmos de propagação têm seu funcionamento centrado em uma série de iterações que objetivam reduzir a taxa de erro das RNAs (Silva et al., 2016). A forma que a taxa de erro é utilizada pela rede e como o gradiente do mesmo é atualizado varia conforme o algoritmo de treinamento implementado (Shrestha \& Mahmood, 2019). Alguns desses algoritmos mais conhecidos são o Backpropagation, Resilient Propagation, Quick Propagation, Scaled Conjugate Gradient e o Manhattan Update Rule.

$\mathrm{Na}$ mensuração florestal, os algoritmos Resilient Propagation e Backpropagation são os mais testados para predições de variáveis (Miguel et al., 2016; Lacerda et al., 2017; Cunha Neto et al., 2021). O Resilient Propagation permite que cada valor de matriz de peso individual seja treinado individualmente (Heaton, 2011). A principal desvantagem deste algoritmo é sua maior complexidade de implementação do que o Backpropagation. Por outro lado, o Backpropagation fornece uma maneira muito simples e eficiente de calcular o gradiente em uma RNA (Chen \& Gu, 2020). Contudo, o Backpropagation leva mais tempo para o treinamento da RNA devido aos movimentos backwards que o neurônio executa até que a solução ideal seja encontrada (Zajmi et al., 2018).

Para os demais algoritmos, algumas das vantagens da utilização do Scaled Conjugate Gradient, Quick propagation e Manhattan Update Rule são, respectivamente, a não utilização de busca unidimensional a cada iteração para determinar a taxa de aprendizagem (Selvamuthu et al., 2019), a desnecessidade de fornecer um valor para o parâmetro momentum e a facilidade no processamento dos dados (Heaton, 2011). Porém, essa simplicidade do algoritmo Manhattan Update Rule pode gerar resultados ligeiramente piores.

Assim, estudos que testam melhores opções na configuração de uma RNA para a predição da altura total das árvores são importantes para aumentar a acurácia das predições (Martins et al., 
2016a). Trabalhamos com a hipótese de que as configurações de redes neurais artificiais influenciam na precisão da predição da altura total das árvores de eucalipto. Neste sentido, o objetivo deste estudo foi recomendar as melhores configurações de RNAs para a predição da altura total das árvores de Eucalyptus spp. na mesorregião sudeste do estado do Pará.

\section{MATERIAL E MÉTODOS}

\section{Descrição da área experimental}

O estudo foi realizado em áreas de plantios comerciais da Fazenda Formosa, localizada no município de Paragominas - PA, mesorregião Sudeste Paraense. A região do estudo possui características homogêneas de paisagem, com solos do tipo Latossolo Amarelo Distrófico, textura muito arenosa e com relevo plano suavemente ondulado (Empresa Brasileira de Pesquisa Agropecuária, 2018). O clima é do tipo "Aw" segundo a classificação climática de Köppen, com umidade relativa do ar por volta de $85 \%$, temperatura média anual de $26^{\circ} \mathrm{C}$ e precipitação pluviométrica média anual entre 1.900 e 2.200 mm.ano-1 (Alvares et al., 2013).

\section{Coleta das variáveis dendrométricas quantitativas e qualitativas}

Os dados foram provenientes de seis clones de Eucalyptus spp., com idades variando de 10 a 59 meses (Tabela 1). Os seis clones estudados foram distribuídos em 40 talhões, variando de 11,5 a 42,8 ha, sendo que nessas áreas foram instaladas parcelas para realização do

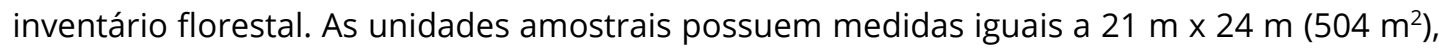
alocadas de forma aleatória na área com intensidade amostral de uma parcela a cada 15 ha. Foram amostradas 2.888 árvores.

Tabela 1. Listagem das espécies e híbridos de Eucalyptus spp. mensurados

\begin{tabular}{ccc}
\hline Clone & Espécie/Híbrido & N \\
\hline C1 & Eucalyptus grandis W. Hill ex Maiden × Eucalyptus sp. & 63 \\
C2 & Eucalyptus grandis W. Hill ex Maiden $\times$ Eucalyptus urophylla S.T. Blake & 71 \\
C3 & Eucalyptus sp. & 558 \\
C4 & Eucalyptus urophylla S.T. Blake $\times$ Eucalyptus grandis W. Hill ex Maiden & 216 \\
C5 & Eucalyptus urophylla S.T. Blake & 763 \\
C6 & Eucalyptus brassiana S.T. Blake $\times$ Eucalyptus grandis W. Hill ex Maiden & 1217 \\
\hline
\end{tabular}

A mensuração das variáveis quantitativas de CAP (circunferência a 1,30 m do solo) para posterior transformação em DAP (diâmetro a 1,30 m do solo), utilizando fita centimétrica, e também mensuração da altura total das árvores, utilizando clinômetro digital Haglof ${ }^{\circledR}$ foram realizadas para cada indivíduo dentro da parcela.

A tabulação dos dados coletados em campo foi realizada para posterior classificação em classes para as variáveis idade e DAP (Tabela 2).

Tabela 2. Estatística descritiva da altura total e das classes de idade e diâmetro a 1,30 m de altura do solo (DAP)

\begin{tabular}{|c|c|c|c|c|c|c|c|}
\hline \multirow{2}{*}{$\begin{array}{l}\text { Estatística } \\
\text { descritiva }\end{array}$} & \multicolumn{3}{|c|}{ Idade (meses) } & \multicolumn{3}{|c|}{ DAP (cm) } & \multirow{2}{*}{ Altura (m) } \\
\hline & Classe 1 & Classe 2 & Classe 3 & Classe 1 & Classe 2 & Classe 3 & \\
\hline Amplitude & $10-26,3$ & $26,4-42,7$ & $42,8-59$ & $2,07-8,8$ & $8,9-15,6$ & $15,7-22,28$ & $2,8-34$ \\
\hline $\mathrm{N}$ & 672 & 711 & 1505 & 592 & 1988 & 308 & 2888 \\
\hline $\bar{x}$ & 23,28 & 31,5 & 54 & 6,47 & 11,77 & 16,81 & 15,19 \\
\hline$s$ & $\pm 5,27$ & $\pm 2,8$ & $\pm 2,7$ & $\pm 1,99$ & $\pm 1,78$ & $\pm 1,06$ & $\pm 4,47$ \\
\hline
\end{tabular}

Em que: $\mathrm{N}=$ número de árvores; $\bar{x}=$ média; $s=$ desvio padrão. 


\section{Treinamento e combinações para a melhor estrutura de RNA}

As RNA treinadas foram do tipo Multilayer Perceptron (MLP), com apenas uma camada oculta. As variáveis qualitativas utilizadas nos treinamentos foram o clone, classe de idade e classe diamétricas. As variáveis quantitativas foram a idade (em meses), DAP (cm). A altura total dos indivíduos foi a variável de saída. Os dados foram divididos para o treinamento (70\%) e validação (30\%) das RNA. O software Neuro 4.0.6 (Binoti, 2012) foi utilizado para configuração, treinamento e validação das mesmas. As variáveis qualitativas foram inseridas no software como fator numerado de 1 a 3, uma vez que o programa permite a diferenciação entre variáveis qualitativas e quantitativas. O número de neurônios na camada oculta variou de 2 a 30. Cinco algoritmos, cinco funções de ativação e todas as possíveis combinações entre eles foram testados.

Os algoritmos de aprendizagem testados foram o Backpropagation, Quick Propagation, Resilient Propagation, Manhattan Update Rule e Scaled Conjugate Gradient. No algoritmo Backpropagation foram configurados auto ajustes nas taxas de aprendizagem e momentum, para reduzir os valores destes parâmetros com a evolução do algoritmo. O algoritmo de treinamento Quick Propagation provém do método de Newton, utilizado para aquisição de raízes de uma função. Sendo esse algoritmo tolerante a altas taxas de aprendizagem, o valor igual a 1 foi utilizado. A variação básica RPROP+ do algoritmo Resilient Propagation foi utilizada para o treinamento. Nesse caso a direção de cada atualização de peso é baseada no sinal da derivada parcial ( $\partial \mathrm{E} / \partial$ wij). Para o algoritmo Manhattan Update Rule, que por definição necessita de baixas taxas de aprendizado, o valor 0,00001 foi utilizado. O algoritmo Scaled Conjugate Gradient não tem variações em sua configuração, sem dependência a valores de critérios para treinamento.

As funções de ativação (FA) utilizadas foram as seguintes: logística (sigmoide), tangente, $\log$, linear e seno. A priori, foram geradas cinco redes para cada topologia, em que cada combinação de funções resultou em 145 redes ( 5 redes $\times 29$ variações de neurônios). Assim, para cada função dentro de um algoritmo foram treinadas 725 RNA, gerando 3.625 redes por algoritmo.

O erro médio $(0,001)$ ou o número de ciclos $(3000)$, com valor para taxa de convergência igual a 20 foram utilizados como critério de parada no treinamento das RNA.

\section{Parâmetros de seleção das melhores redes neurais artificiais}

As redes neurais artificiais foram avaliadas segundo três critérios: o bias (Equação 1), a raiz quadrada do erro médio (RQEM\%) (Equação 2), coeficiente de correlação de Pearson entre altura total observada e altura total predita pela RNA $\left(r_{\hat{y y y}}\right)$ (Equação 3). As medidas de $r_{\hat{y} y}$, bias e de RQEM\% para as 18.125 redes treinadas foram separadas em classes (com intervalos de 0,02 para $r_{\hat{y} y}, 5 \%$ e $10 \%$ para o RQEM\%) e utilizadas na geração de histogramas por algoritmos e funções de ativação.

$$
\text { bias }=\frac{\sum_{i=1}^{n}\left(\hat{y}_{i}-y_{i}\right)}{n}
$$

$$
\begin{aligned}
& R Q E M \%=\frac{100}{\bar{y}} \cdot \sqrt{\frac{\sum_{i=1}^{n}\left(\hat{y}_{i}-y_{i}\right)^{2}}{n}} \\
& r_{\hat{y} y}=\frac{n^{-1} \sum_{i=1}^{n}\left(\hat{y}_{i}-\hat{y}_{m}\right)\left(y_{i}-\bar{y}\right)}{\sqrt{n^{-1} \sum_{i=1}^{n}\left(\hat{y}_{i}-\hat{y}_{m}\right)^{2} n^{-1} \sum_{i=1}^{n}\left(y_{i}-\bar{y}\right)^{2}}}
\end{aligned}
$$


Em que: $\hat{y}_{i}=$ altura total predita de cada observação $(\mathrm{m}) ; y_{i}=$ altura total observada de cada observação $(\mathrm{m}) ; n$ = número de observações; $\bar{y}=$ média das alturas totais observadas $(\mathrm{m})$; $\hat{y}_{m}=$ média das alturas totais preditas $(\mathrm{m})$.

\section{RESULTADOS E DISCUSSÃO}

Dentre as 18.125 redes treinadas, sendo 3.625 por algoritmo, um total de 658 redes não convergiram. Os algoritmos Quick Propagation, Resilient Propagation e Scaled Conjugate Gradient alcançaram mais de $96 \%$ de convergência das redes treinadas. O algoritmo Manhattan Update Rule obteve $100 \%$ de convergência. Em contrapartida, o algoritmo Backpropagation apresentou $440(12,13 \%)$ redes que não convergiram.

Na Figura 1 estão apresentados a frequência por classe das métricas de $r_{\hat{y} y}$ e RQEM\% das RNA treinadas que convergiram para cada algoritmo. O algoritmo Manhattan Update Rule obteve mais de $97 \%$ das redes com $r_{\hat{y} y}$ menor que 0,60 e $27,8 \%$ com $r_{\hat{y} y}$ negativa. Os valores de RQEM\% das redes treinadas com este algoritmo variaram de $10 \%$ a $400 \%$.

O algoritmo Manhattan Update Rule obteve maior taxa de convergência e menor precisão das estimativas devido a sua simplicidade no treinamento. Esse algoritmo usa apenas o sinal do gradiente na atualização dos pesos, desconsiderando sua magnitude. Além de permitir a atualização dos pesos em paralelo, reduzindo o número de ciclos no processo de treinamento (Zamanidoost et al., 2015). Essa forma de atualização dos pesos pode dificultar a alcançar um erro mínimo global, gerando redes com resultados insatisfatórios.
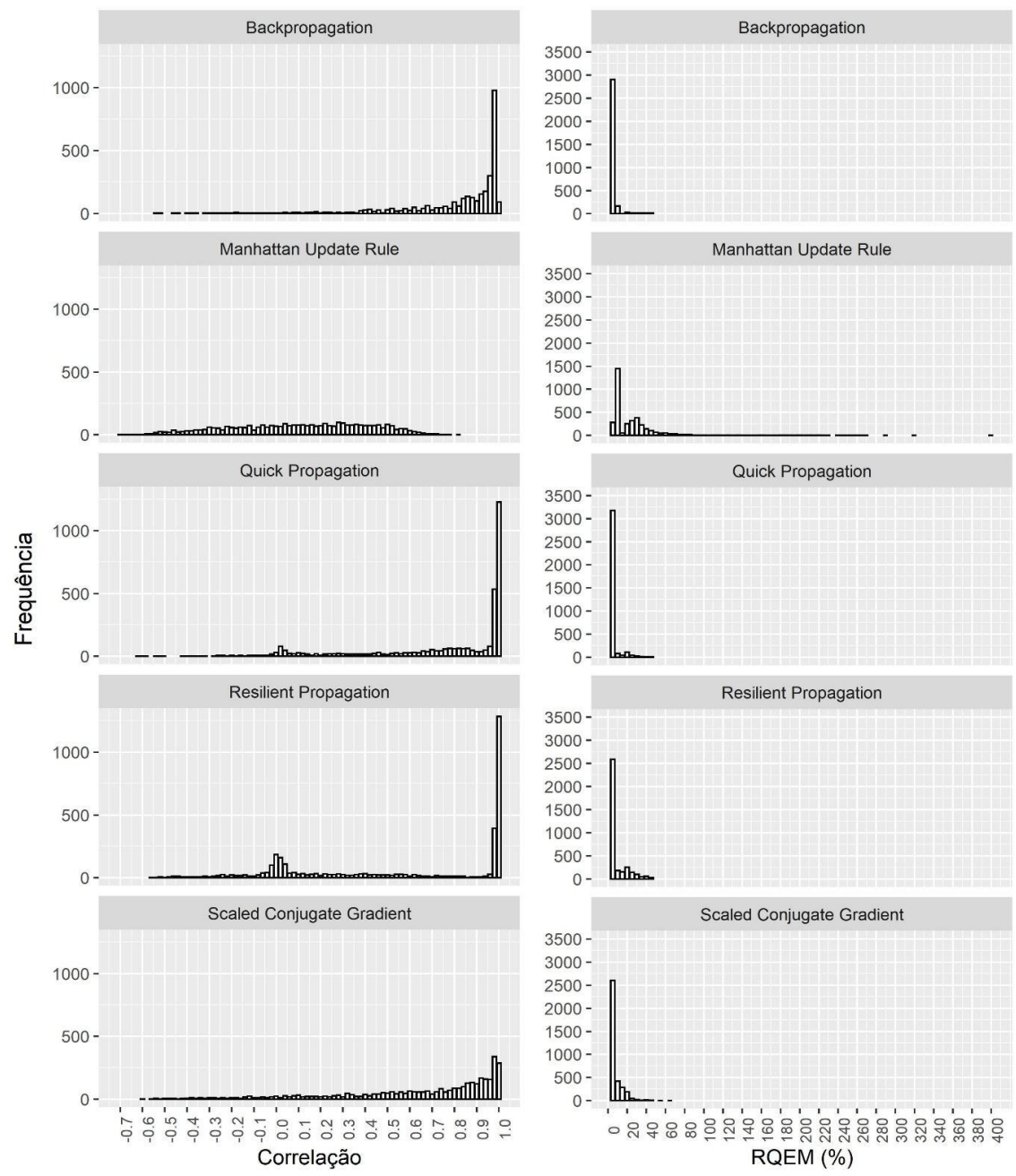

Figura 1: Frequência por classe das métricas de correlação e RQEM\% das redes neurais artificiais treinadas para cada algoritmo. 
Os algoritmos Backpropagation, Resilient Propagation e Quick Propagation alcançaram $r_{\hat{y} y}$ maior que 0,95 em 42, 49 e 51\% das redes, respectivamente. Esses algoritmos obtiveram um RQEM\% menor que 5\% em mais de 91, 73 e 90\% das redes treinadas, respectivamente. $O$ algoritmo Scaled Conjugate Gradient apresentou $20 \%$ das redes com $r_{\hat{y} y}$ maior que 0,95. Além disso, obteve um RQEM\% menor que $5 \%$ em $72 \%$ dos dados, com uma variação de 5 a $65 \%$ no restante das redes.

O bom desempenho do Backpropagation pode ser explicado pela robustez do algoritmo com muitos parâmetros que otimiza a modelagem e reduz os erros, como por exemplo a taxa de aprendizado e momentum (Heaton, 2011; Shamsuddin et al., 2013; Miguez et al., 2014; Zamanidoost et al., 2015). O resultado satisfatório do algoritmo Quick Propagation pode estar relacionado à sua tolerância a taxas de aprendizagem mais altas, sendo possível obter redes mais precisas (Heaton, 2011).

Os algoritmos Resilient Propagation e Scaled Conjugate Gradient obtiveram redes com boa precisão nas estimativas devido à maior flexibilidade durante o treinamento. Neste caso, os parâmetros não precisam ser definidos previamente, sendo assim o próprio algoritmo define os valores ideais para os parâmetros (Heaton, 2011; Martins et al., 2016b).

Em relação aos histogramas de frequência para as FA utilizadas na camada oculta (Figura 2), a função de ativação linear resultou em RNA com maior incidência de $r_{\hat{y} y}$ negativa (19\%) e presença de redes com RQEM\% superiores a 100\% (2,9\%). A função de ativação do tipo logística apresentou a menor quantidade de redes treinadas com coeficientes de $r_{\hat{y} y}$ negativos (3,4\%), e nenhuma RNA com RQEM\% superior a 100\%.
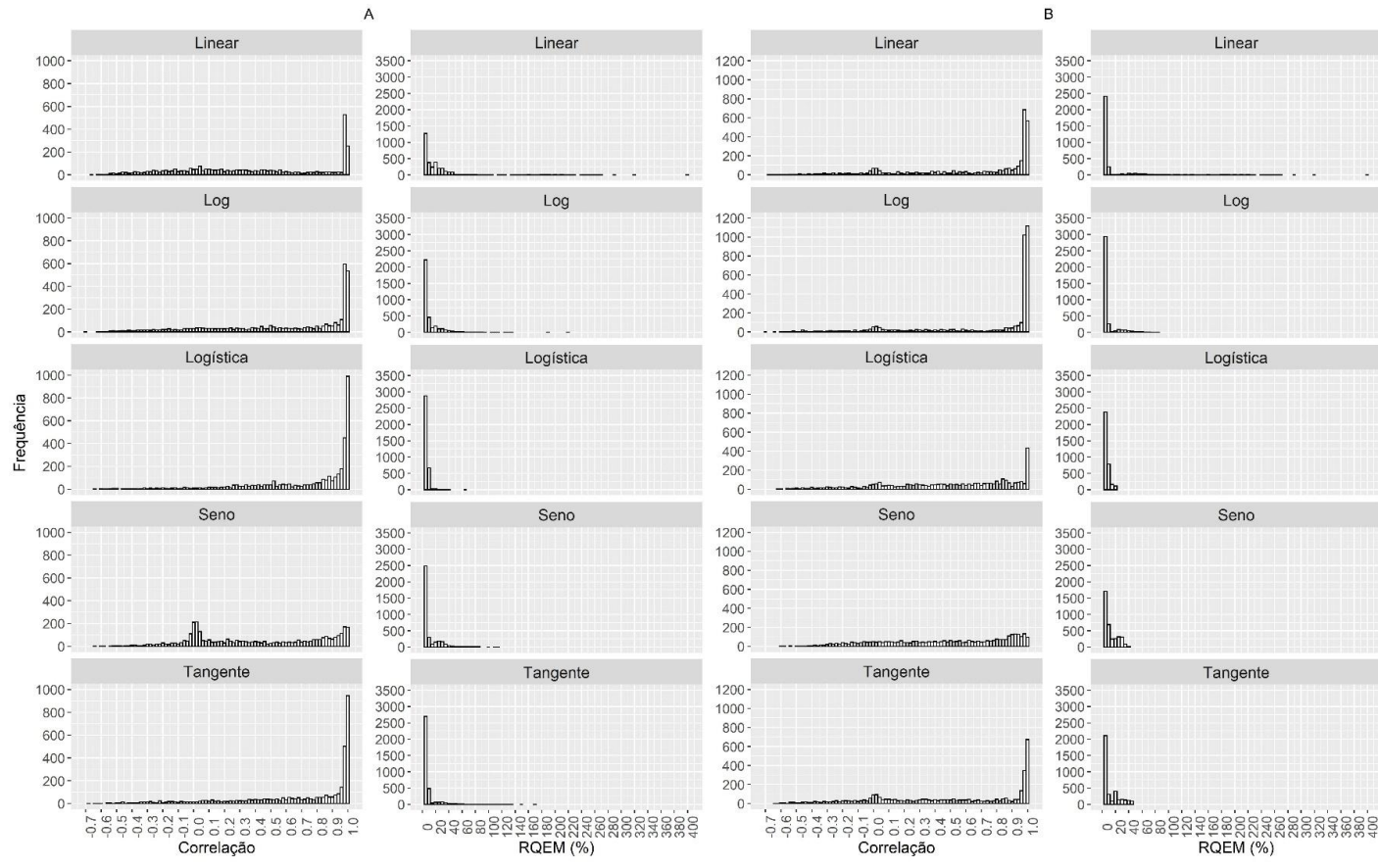

Figura 2. Frequência por classe das métricas de correlação e RQEM\% para as funções de ativação na camada oculta (A) e função de ativação na camada de saída (B).

Ao analisar os histogramas das FA para a camada de saída (Figura 2), as funções tangente e seno obtiveram as maiores frequências de RNA com $r_{\hat{y} y}$ negativas $(11,9 \%$ e $10,9 \%$, respectivamente) e as menores concentrações de RNA com RQEM\% inferiores a 10\%. Para a função log, $89 \%$ das redes apresentaram erros inferiores a $10 \%$ e somente $6 \%$ das redes obtiveram $r_{\hat{y} y}$ negativas.

As diferenças nas distribuições de frequência das métricas entre as funções de ativação indicam haver uma contribuição relevante dessas para as predições da altura total de Eucalyptus spp. Desse modo, a função de ativação influencia de forma significativa a complexidade da 
aprendizagem e o desempenho de uma rede neural artificial, uma vez que é o componente responsável por introduzir não-linearidade na RNA (Lecun et al., 2015; Mohammed et al., 2019).

A função do tipo linear demonstrou desempenho inferior na camada oculta e de saída, indicado por grandes quantidades de redes treinadas com baixas $r_{\hat{y} y}$. A perda de precisão com a utilização de função linear nas camadas ocultas das redes está associada às relações não-lineares existentes entre as variáveis utilizadas para a predição da altura das árvores (Martins et al., 2016a). As topologias implementadas com função de ativação do tipo logística na camada oculta, resultam em redes com frequências das métricas de precisão mais adequadas. O melhor desempenho desta função pode estar associado ao seu balanço, entre linearidade e não linearidade, que permite a boa representação de fenômenos não-lineares em casos de aproximação de funções para predições de variáveis dendrométricas (Binoti et al., 2014; Lopes et al., 2020).

A função de ativação tangente é amplamente utilizada na implementação de redes neurais artificiais para aproximação de funções, por conta do seu intervalo mais flexível, variando de -1 a 1 (Hajduk, 2017). No entanto, gerou um elevado número de redes com $r_{\hat{y} y}$ negativas e altos RQEM\% na camada de saída. Esse desempenho inferior pode ter sido ocasionado pelas diferentes combinações entre funções de ativação das camadas ocultas e de saída. Se utilizada em ambas as camadas, a função de ativação tangente é capaz de predizer com boa precisão variáveis dendrométricas (Martins et al., 2015; Martins et al., 2016a).

As menores quantidades de redes com $r_{\hat{y} y}$ negativas foram obtidas com adoção da função de ativação log na camada de saída, o que indica bom desempenho das topologias dotadas com esta função. Devido à sua capacidade de lidar com nós saturados que retardam o treinamento, a utilização da função log é recomendada para casos em que o treinamento de redes com função tangente não é bem sucedido (Heaton, 2011).

A frequência de redes em cada classe de $r_{\hat{y} y}$ e RQEM\% variou entre as combinações de algoritmos e funções de ativação (Figura 3 e Figura 4). Os algoritmos Quick Propagation e Backpropagation obtiveram maiores frequências de redes com estatísticas de precisão elevadas, mesmo quando utilizando funções de ativação que tenderam a gerar redes com menores estatísticas de precisão (linear, seno, tangente e logística). O algoritmo Manhattan Update Rule apresentou maior quantidade de redes com baixa precisão, em todas as funções de ativação.
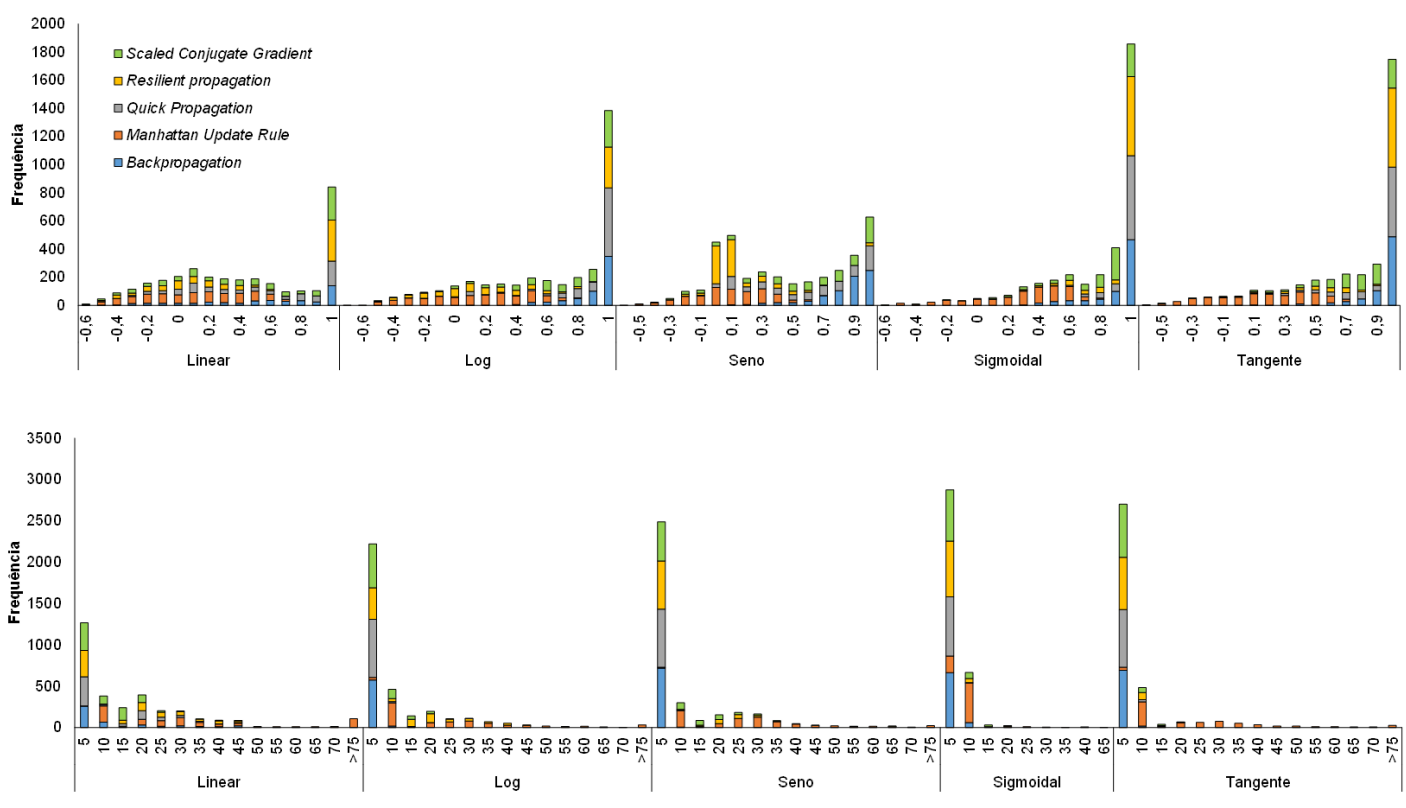

Figura 3: Frequência de redes em cada classe de correlação e RQEM\% entre as combinações de algoritmos e funções de ativação na camada oculta. 

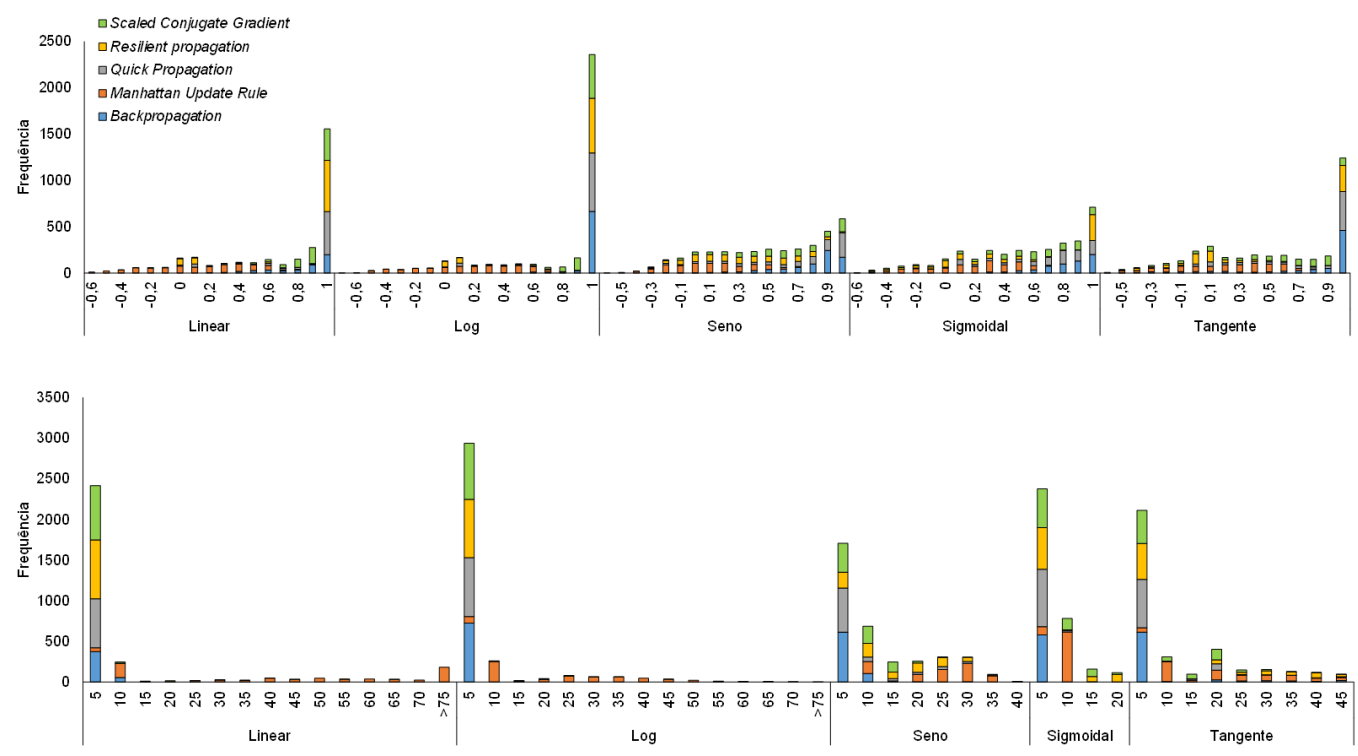

Figura 4: Frequência de redes em cada classe de correlação e RQEM\% entre as combinações de algoritmos e funções de ativação na camada de saída.

A variação da frequência de redes em cada classe de $r_{\hat{y y y}}$ e RQEM\% a partir da combinação de algoritmos e funções de ativação sugere que algoritmos com processos de treinamento mais robustos podem apresentar bons desempenhos mesmo utilizando combinações de funções (camada oculta e de saída) menos eficientes (Gupta et al., 2020).

As topologias de RNA com algoritmo Resilient Propagation apresentaram uma participação expressiva na classe de correlação $>0,90$ em todas as funções de ativação, com exceção da função seno que obteve redes com menores $r_{\hat{y} y}$ e maiores RQEM\%.

O baixo desempenho estatístico do algoritmo Resilient Propagation combinado com a função seno indica que essa função tem efeito direto no tempo de treinamento e desempenho desse algoritmo, uma vez que se relacionam com o processo de atualização dos pesos durante a etapa de treinamento das redes (Miguez et al., 2014).

Em relação às melhores RNA selecionadas em função das estatísticas de validação, a $r_{\hat{y} y}$ apresentou valores acima de 0,97, e RQEM e bias próximos de zero para a maioria dos algoritmos (Tabela 3). $\mathrm{O}$ algoritmo Manhattan Update Rule foi a exceção, apresentando $r_{\hat{y} y}$ igual a 0,80, e RQEM e bias maiores em comparação aos outros algoritmos. A topologia de rede 1210-1, com algoritmo Resilient Propagation e função de ativação logística na camada oculta e de saída apresentou as melhores estatísticas, sendo essa a melhor RNA encontrada. O número de neurônios na camada oculta da maioria das melhores RNA foi igual ou superior a 10, com exceção da RNA com algoritmo Quick Propagation que apresentou apenas 2 neurônios.

Tabela 3. Configurações e estatísticas de precisão das melhores RNA na validação

\begin{tabular}{ccccccc}
\hline Algoritmo & CO & CS & Topologia & $r_{\hat{y} y}$ & Bias & RQEM \\
\hline Backpropagation & Tangente & Log & $12-23-1$ & 0,97504 & 0,0030 & 0,7637 \\
Quick propagation & Tangente & Tangente & $12-2-1$ & 0,99798 & $-0,0039$ & 0,2436 \\
Resilient propagation & Logística & Logística & $12-10-1$ & 0,99989 & $-0,0007$ & 0,0670 \\
Manhattan Update Rule & Tangente & Log & $12-14-1$ & 0,80275 & 0,2607 & 3,1315 \\
Scaled Conjugate Gradient & Log & Log & $12-30-1$ & 0,9994 & $-0,0005$ & 0,1634 \\
\hline
\end{tabular}

Em que: CO: camada oculta; CS: camada de saída; $r_{\hat{y} y}$ : coeficiente de correlação de Pearson entre altura total observada e altura total predita pela RNA; RQEM: raiz quadrada do erro médio. 
Em relação às estatísticas de validação (Tabela 3), a maior precisão da RNA com o algoritmo Resilient Propagation combinado com a função logística nas duas últimas camadas comprova que esse algoritmo e essa função apresentam grande aptidão para a aplicação em variáveis florestais (Leite et al., 2016; Wang et al., 2017; Tavares Júnior et al., 2020). Entretanto, o bom desempenho estatístico dos demais algoritmos, com exceção do Manhattan Update Rule, comprova que esses algoritmos também podem gerar predições com grande precisão.

A alta precisão da RNA com a função logística combinada com o algoritmo Resilient Propagation indica que testar as interações entre o algoritmo e a função de ativação pode resultar no aumento da precisão da predição. A presença da função log na RNA com o algoritmo Backpropagation (> precisão) e na RNA com o algoritmo Manhattan Update Rule (< precisão) reforça a importância de se testar as configurações mais adequadas para cada tipo de problema (Raghu \& Sriraam, 2017; Naik et al., 2020).

Já em relação aos neurônios na camada oculta, o número de neurônios igual ou superior a 10 para a maioria das RNA está relacionado com a complexidade do problema apresentado a RNA, em que quanto maior a quantidade de informação apresentada para a rede, maior será a quantidade de neurônios necessários para resolver o problema (Binoti et al., 2014). Esse número foi maior que o encontrado em outros estudos, em que apresentaram de um a quatro neurônios nessa camada (Binoti et al., 2014; Campos et al., 2016).

\section{CONCLUSÃO}

Os algoritmos Backpropagation, Resilient Propagation, Scaled Conjugate Gradient e Quick Propagation são eficientes na predição da altura total de Eucalyptus spp. Entretanto, o algoritmo Manhattan Update Rule não é adequado para essa aplicação.

As funções de ativação logística e log são as mais eficientes para as camadas oculta e de saída, respectivamente. Por outro lado, a função linear na camada oculta e tangente na camada de saída não geram redes com boa precisão.

A topologia de rede 12-10-1, com algoritmo Resilient Propagation e função de ativação logística na camada oculta e de saída pode ser utilizada no treinamento para a predição da altura total de Eucalyptus spp.

\section{AGRADECIMENTOS}

Ao Conselho Nacional de Desenvolvimento Científico e Tecnológico (CNPq) e a Coordenação de Aperfeiçoamento de Pessoal de Nível Superior (CAPES) - código de financiamento 001.

\section{REFERÊNCIAS BIBLIOGRÁFICAS}

Alvares, C. A., Stape, J. L., Sentelhas, P. C., de Moraes Gonçalves, J. L., \& Sparovek, G. (2013). Köppen's climate classification map for Brazil. Meteorologische Zeitschrift (Berlin), 22(6), 711-728. http://dx.doi.org/10.1127/0941-2948/2013/0507.

Binoti, M. L. M. S. (2012). Redes neurais artificiais em mensuração e manejo florestal (Tese de doutorado). Universidade Federal de Viçosa, Viçosa, MG.

Binoti, D. H. B., Binoti, M. L. M. S., \& Leite, H. G. (2014). Configuração de redes neurais artificiais para estimação do volume de árvores. Ciência da Madeira, 5(1), 58-67. http://dx.doi.org/10.12953/21776830.v05n01a06.

Binoti, D. H. B., Binoti, M. L. M. S., Leite, H. G., \& Silva, A. (2013). Redução dos custos em inventário de povoamentos equiâneos. Agrária, 08(01), 125-129. http://dx.doi.org/10.5039/agraria.v8i1a2209.

Campos, B. P. F., Silva, G. F., Binoti, D. H. B., Mendonça, A. R., \& Leite, H. G. (2016). Predição da altura total de árvores em plantios de diferentes espécies por meio de redes neurais artificiais. Pesquisa Florestal Brasileira, 36(88), 375-385. http://dx.doi.org/10.4336/2016.pfb.36.88.1166.

Campos, J. C. C., \& Leite, H. G. (2017). Mensuração florestal: perguntas e respostas (5. ed.). Viçosa: Editora UFV. 636 p. 
Chen, C. T., \& Gu, G. X. (2020). Generative deep neural networks for inverse materials design using backpropagation and active learning. Advancement of Science, 7(5), 1-10. PMid:32154072. http://dx.doi.org/10.1002/advs.201902607.

Cunha Neto, E. M., Moura, M. M., Araujo, E. C. G., Santana, G. M., Corte, A. P. D., \& Sanquetta, C. R. (2021). Aprendizado de máquina e regressão linear na estimativa do volume de Eucalyptus na Amazônia Oriental. BIOFIX Scientific Journal, 6(1), 08-14.

Cysneiros, V. C., Pelissari, A. L., Gaui, T. D., Fiorentin, L. D., Carvalho, D. C., Silveira Filho, T. B., \& Machado, S. A. (2020). Modeling of tree height-diameter relationships in the Atlantic Forest: effect of forest type on tree allometry. Canadian Journal of Forest Research, 50(12), 1289-1298. http://dx.doi.org/10.1139/cjfr-2020-0060.

Demertzis, K., Iliadis, L., Avramidis, S., \& El-Kassaby, Y. A. (2017). Machine learning use in predicting interior spruce wood density utilizing progeny test information. Neural Computing \& Applications, 28(3), 505-519. http://dx.doi.org/10.1007/s00521-015-2075-9.

Empresa Brasileira de Pesquisa Agropecuária - EMBRAPA. (2018). Sistema brasileiro de classificação de solos (5. ed.). Brasília, DF: EMBRAPA. 356p.

Gupta, N., Bedi, P., \& Jindal, V. (2020). Effect of activation functions on the performance of deep learning algorithms for network intrusion detection systems. In P. K. Singh, B. K. Panigrahi, N. K. Suryadevara, S. K. Sharma, A. P. Singh. Proceedings of ICETIT 2019 (pp. 949-960). Delhi: Springer. http://dx.doi.org/10.1007/978-3-030-30577-2_84.

Hajduk, Z. (2017). High accuracy FPGA activation function implementation for neural networks. Neurocomputing, 247, 59-61. http://dx.doi.org/10.1016/j.neucom.2017.03.044.

Heaton, J. (2011). Programming Neural Networks with Encog3 in Java (2nd ed., 240 p.). Heaton Research, Incorporated.

Lacerda, T. H. S., Cabacinha, C. D., Araújo Júnior, C. A., Maia, R. D., \& Lacerda, K. W. (2017). Redes neurais artificiais para estimar o volume de árvores no cerrado. Cerne, 23(4), 483-491. http://dx.doi.org/10.1590/01047760201723042347.

Lecun, Y., Bengio, Y., \& Hinton, G. (2015). Deep learning. Nature, 521(7553), 436-444. PMid:26017442. http://dx.doi.org/10.1038/nature14539.

Leite, H. G., Binoti, D. H. B., Oliveira Neto, R. R., Lopes, P. F., Castro, R. R., Paulino, E. J., Binoti, M. L. M. S., \& Colodette, J. L. (2016). Redes Neurais Artificiais para a estimação da densidade básica da madeira. Scientia Florestalis, 44(109), 149-154.

Lopes, L. S. S., Rode. R., Pauletto, D., Baloneque, D. D., Santos, F. G., Silva, A. R., Binoti, D. H. B., \& Leite, H. G. (2020). Uso de regressão e redes neurais artificiais na estimativa do volume de Khaya ivorensis. Revista Ciência da Madeira, 11(2), 74-84.

Martins, E. R., Binoti, M. L. M. S., Leite, H. G., Binoti, D. H. B., \& Dutra, G. C. (2015). Configuração de redes neurais artificiais para prognose da produção de povoamentos clonais de eucalipto. Agrária, 10(4), 532-537. http://dx.doi.org/10.5039/agraria.v10i4a5350.

Martins, E. R., Binoti, M. L. M. S., Leite, H. G., Binoti, D. H. B., \& Dutra, G. C. (2016a). Configuração de redes neurais artificiais para estimação da altura total de árvores de eucalipto. Agrária, 11(2), 117123. http://dx.doi.org/10.5039/agraria.v11i2a5373.

Martins, E. R., Binoti, M. L. M. S., Leite, H. G., Binoti, D. H. B., \& Dutra, G. C. (2016b). Configuração de redes neurais artificiais para estimação do afilamento do fuste de árvores de eucalipto. Agrária, 11(1), 33-38. http://dx.doi.org/10.5039/agraria.v11i1a5354.

Melo, E. A., Calegario, N., Mendonça, A. R., Possato, E. L., Alves, J. A., \& Isaac Júnior, M. A. (2017). Modelagem não linear da relação hipsométrica e do crescimento das árvores dominantes e codominantes de Eucalyptus sp. Ciência Florestal, 27(4), 1325-1338. http://dx.doi.org/10.5902/1980509829895.

Miguel, E. P., Mota, F. C. M., Téo, S. J., Nascimento, R. G. M., Leal, F. A., Pereira, R. S., \& Rezende, A. V. (2016). Artificial intelligence tools in predicting the volume of trees within a forest stand. African Journal of Agricultural Research, 11(21), 1914-1923. http://dx.doi.org/10.5897/AJAR2016.11015.

Miguez, G., Xavier, A. E., \& Maculan, N. (2014). An evaluation of the bihyperbolic function in the optimization of the backpropagation algorithm. International Transactions in Operational Research, 21(5), 835-854. http://dx.doi.org/10.1111/itor.12072.

Mohammed, M. A., Naji, T. A., \& Abduljabbar, H. M. (2019). The effect of the activation functions on the classification accuracy of satellite image by artificial neural network. Energy Procedia, 157, 164-170. http://dx.doi.org/10.1016/j.egypro.2018.11.177. 
Naik, D. L., Sajid, H. U., Kiran, R., \& Chen, G. (2020). Detection of corrosion-indicating oxidation product colors in steel bridges under varying illuminations, shadows, and wetting conditions. Metals, 10(11), 1-19. http://dx.doi.org/10.3390/met10111439.

Nandy, S., Singh, R., Ghosh, S., Watham, T., Kushwaha, S. P. S., Kumar, A. S., \& Dadhwal, V. K. (2017). Neural network-based modelling for forest biomass assessment. Carbon Management, 8(4), 305317. http://dx.doi.org/10.1080/17583004.2017.1357402.

Özçelik, R., Diamantopoulou, M. J., Brooks, J. R., \& Wiant Junior, H. V. (2010). Estimating tree bole volume using artificial neural network models for four species in Turkey. Journal of Environmental Management, 91(3), 742-753. PMid:19880241. http://dx.doi.org/10.1016/j.jenvman.2009.10.002.

Raghu, S., \& Sriraam, N. (2017). Optimal configuration of multilayer perceptron neural network classifier for recognition of intracranial epileptic seizures. Expert Systems with Applications, 89, 205-221. http://dx.doi.org/10.1016/j.eswa.2017.07.029.

Selvamuthu, D., Kumar, V., \& Mishra, A. (2019). Indian stock market prediction using artificial neural networks on tick data. Financial Innovation, 5(1), 1-12.

Shamsuddin, S. M., Ibrahim, A. O., \& Ramadhena, C. (2013). Weight changes for learning mechanisms in two-term back-propagation network. In Suzuki, K. Artificial neural networks - Architectures and applications (pp. 53-81).New York: InTech.

Shrestha, A., \& Mahmood, A. (2019). Review of deep learning algorithms and architectures. IEEE Access: Practical Innovations, Open Solutions, 7, 53040-53065. http://dx.doi.org/10.1109/ACCESS.2019.2912200.

Silva, I. N., Spatti, D. H., Flauzino, R. A., Liboni, L. H. B., \& Dos Reis Alves, S. F. (2016). Artificial neural networks: A practical course (p. 1-307). Springer International Publishing.

Tavares Júnior, I., Torres, C. M. M. E., Leite, H. G., Castro, N. L. M., Soares, C. P. B., Castro, R. V. O., \& Farias, A. A. (2020). Machine learning: modeling increment in diameter of individual trees on Atlantic Forest fragments. Ecological Indicators, 117, 106685. http://dx.doi.org/10.1016/j.ecolind.2020.106685.

Vendruscolo, D. G. S., Chaves, A. G. S., Medeiros, R. A., Silva, R. D., Souza, H. S., Drescher, R., \& Leite, H. G. (2017). Estimativa da altura de árvores de Tectona grandis Lf utilizando regressão e redes neurais artificiais. Nativa (Sinop), 5(1), 52-58. http://dx.doi.org/10.5935/2318-7670.v05n01a09.

Vieira, G. C., Mendonça, A. R., Silva, G. F., Zanetti, S. S., Silva, M. M., \& Santos, A. R. (2018). Prognoses of diameter and height of trees of eucalyptus using artificial intelligence. The Science of the Total Environment, 619-620, 1473-1481. PMid:29734623. http://dx.doi.org/10.1016/j.scitotenv.2017.11.138.

Wang, L., Liu, J., Xu, S., Dong, J., \& Yang, Y. (2017). Forest above ground biomass estimation from remotely sensed imagery in the mount tai area using the RBF ann algorithm. Intelligent Automation and Soft Computing, 24(2), 391-398. http://dx.doi.org/10.1080/10798587.2017.1296660.

Zajmi, L., Ahmed, F. Y. H., \& Jaharadak, A. A. (2018). Concepts, methods, and performances of particle swarm optimization, backpropagation, and neural networks. Applied Computational Intelligence and Soft Computing, 2018, 1-7. http://dx.doi.org/10.1155/2018/9547212.

Zamanidoost, E., Bayat, F. M., Strukov, D., \& Kataeva, I. (2015). Manhattan rule training for memristive crossbar circuit pattern classifiers. In: 2015 IEEE 9th International Symposium on Intelligent Signal Processing (WISP) Proceedings (pp. 1-6). Siena, Italy: IEEE. http://dx.doi.org/10.1109/WISP.2015.7139171.

Contribuição dos Autores: JECR: Conceituação, Supervisão, Escrita - Primeira Redação, Validação, Metodologia; MRNJ: Conceituação, Escrita - Primeira Redação, Validação, Metodologia; ISTJ e LSSL: Análise Formal, Metodologia, Escrita - Revisão e Edição, Software; JRMS: Análise Formal, Metodologia, Escrita - Revisão e Edição; MLS: Escrita Revisão e Edição, Validação. 